\title{
Los miembros de los grupos de trabajo del proyecto WFME-Estándares globales
}

Los miembros de los tres grupos de trabajo de la WFME que tratan sobre la Educación Médica Básica, la Educación Médica Postgraduada y el Desarrollo Profesional Continuado de los médicos, se presentan conjuntamente en una lista única. Algunos de ellos han participado en más de un grupo de trabajo. Además, la tarea de producir la trilogía de los Estándares en Educación Médica de la WFME se ha de visualizar como un proceso dinámico que se ha ir construyendo sobre los resultados producidos por los grupos de trabajo anteriores.

Se debe destacar que el desarrollo de la trilogía también se ha beneficiado de otras contribuciones importantes. Estas contribuciones han consistido en un gran número de comentarios escritos y orales, así como discusiones en conferencias y reuniones de ámbito nacional e internacional.

\author{
Dr. Palitha Abeykoon \\ Regional Adviser \\ Human Resources for Health \\ World Health Organization \\ Regional Office for South-East Asia \\ New Delhi, India

\section{Professor A. d'Almeida \\ Director \\ Institut Régional de Santé Publique \\ Université Nationale du Bénin \\ Cotonou, Benin}

\section{Dr. George A.O. Alleyne \\ Regional Director \\ World Health Organization \\ Pan American Health Organization \\ Washington, D.C, USA}

\section{Professor A. P. R. Aluwihare}

University of Peradenyia

Peradenyia, Sri Lanka

\section{Dr. Wolfram Antepohl}

Linköping University Hospital

Linköping, Sweden

\author{
Judith S. Armbruster \\ Executive Director \\ Accreditation Council for Graduate \\ Medical \\ Education (ACGME) \\ Chicago, USA
}

Professor Raja Bandaranayake

Arabian Gulf University

Manama, Bahrain

\author{
Ass. Professor Philip G. Bashook \\ University of Illinois at Chicago \\ Chicago, USA \\ Professor Ralph Bloch \\ Universität Bern \\ Bern, Switzerland \\ Professor Cheng Bo-Ji \\ Peking Medical University \\ Beijing, P.R. China
}

\section{Åse Brinchmann-Hansen}

The Norwegian Medical Association

Oslo, Norway

\section{Professor J. D. Chiphangwi \\ College of Medicine \\ Blantyre, Malawi}

Leif Christensen MSc. Soc.

World Federation for Medical Education

Copenhagen, Denmark

\section{Professor Colin Coles \\ King Alfred's College \\ Winchester, United Kingdom}

\section{Professor Alejandro Cravioto}

President, Panamerican Federation of Associations of Medical Schools (PAFAMS)

Universidad Nacional Autonoma

de Mexico

Mexico, D.F., Mexico
Dr. W. Dale Dauphinee

Executive Director

Medical Council of Canada

Ottawa, Canada

Professor Florian Eitel

Ludwig-Maximilians-

Universität München

Munich, Germany

Professor Charles E. Engel

Centre for Higher Education Studies

University of London

London, United Kingdom

\section{Ms. Mette Fisker}

Business Support Manager

Pfizer Danmark

Copenhagen, Denmark

\section{Dr. Tsuguya Fukui}

Kyoto University

Kyoto City - Japan

Dr. Milagros Garcia-Barbero

World Health Organization

European Center for Integrated

Health Care Services

Barcelona, Spain

\section{Dr. Nancy Gary}

Past President, Educational

Commission for Foreign

Medical Graduates (ECFMG)

Washington, D.C., USA 
Professor Laurie Geffen

President, Association for Medical

Education inWestern Pacific Region (AMEWPR)

The University of Queensland

Herston, Australia

Dr. Hussein A. Gezairy

Regional Director

World Health Organization

Regional Office for the Eastern

Mediterranean

Cairo, Egypt

Professor Ernst Goldschmidt

Copenhagen, Denmark

Professor Janet Grant

Open University Centre for Education in Medicine

Milton Keynes, United Kingdom

\section{Professor André Gouazé}

Conference Internationale des Doyens des Faculté de Medicine d'Expression

Francaise (CIDMEF)

Cedex, France

\section{Professor Enrique Guntsche}

Universidad Nacional de Cuyo

Mendoza, Argentina

\section{Dr. James A. Hallock}

President, Educational Commission

for Foreign

Medical Graduates (ECFMG)

Philadelphia, USA

\section{Professor Hossam Hamdy}

Arabian Gulf University

Manama, Bahrain

\section{Professor John D. Hamilton}

University of Newcastle upon Tyne

Newcastle, United Kingdom

\section{Professor Ronald Harden}

Association for Medical Education

in Europe (AMEE)

University of Dundee

Dundee, United Kingdom
Professor Ian R. Hart

University of Ottawa

Ottawa, Canada

Dr. Hans Asbjørn Holm

Norwegian Medical Association

Oslo, Norway

Dr. Saichi Hosoda

Sakakibara Heart Institute

Tokyo, Japan

\section{Dr. Delon Human}

Secretary General, World Medical

Association (WMA)

Cedex, France

\section{Professor Vincent Hunt}

Brown University

School of Medicine

Rhode Island, USA

Dr. Jens Winther Jensen

Permanent Working Group

of European Junior

Doctors (PWG)

Copenhagen, Denmark

\section{Dr. Moufid Jokhadar}

Arab Board of Medical Specialisations

Damascus University

Damascus, Syria

\section{Professor Abraham Joseph}

Christian Medical College

Vellore, India

\section{Dr. Hans Karle}

President, World Federation

for Medical Education

Copenhagen, Denmark

Dr. Donald G. Kassebaum

Past Vice President

Association of American Medical

Colleges

Gleneden Beach, Oregon, USA

Dr. Shamsh Kassim-Lakha

President, The Aga Khan University

Karachi, Pakistan

\section{Mrs Lorraine Kerse}

Regional Adviser

Human Resources for Health

World Health Organization

Regional Office for the

Western Pacific

Manila, The Philippines

\section{Professor Yong Il Kim}

Past President, Association

for Medical Education in

Western Pacific Region (AMEWPR)

National Teacher Training Center for

Health Personnel

Seoul, South Korea

\section{Dr. Jana Krejcikova}

Institute for Postgraduate Medical

Education

Prague, Czech Republic

\section{Dr. David Leach}

Executive Director

Accreditation Council for Graduate

Medical

Education (ACGME)

Chicago, USA

\section{Professor J.C.K. Lee}

Dean, The Faculty of Medicine

The Chinese University of Hong Kong

Hong Kong, P.R. China

\section{Ass. Professor Stefan Lindgren \\ Lund University \\ Lund, Sweden}

\section{Professor Zhao-feng Lu}

Peking University Health Sciences

Center

Beijing, P.R. China

\section{Professor Oleg S. Medvedev}

Dean, Moscow State University

Moscow, Russian Federation

\section{Dr. Donald E. Melnick}

President, National Board of Medical

Examiners (NBME)

Philadelphia, USA 
Professor Jadwiga Mirecka

Jagiellonian University Medical

School

Krakow, Poland

\section{Dr. Mora-Carrasco}

Universidad Autónoma Xochimilco

Mexico, D.F., Mexico

Professor J.P. de V. van Niekerk

President, Association of Medical

Schools in Africa (AMSA) University

of Cape Town

Cape Town, South Africa

\section{Dr. Jørgen Nystrup}

Past President, Association for Medical Education in Europe (AMEE)

World Federation for Medical

Education (WFME)

Copenhagen, Denmark

\section{Professor Albert Oriol-Bosch}

Institut d'ESTUDIS de la SALUT

Barcelona, Spain

\section{Dr. John Parboosingh}

Royal College of Physicians

and Surgeons of Canada

Alberta, Canada

\section{Dr. José Patinõ}

Executive Director

Panamerican Federation of

Associations of Medical Schools

(PAFAMS)

Bogota, Colombia

\section{Dr. Gregory Paulos}

American Medical Association

Chicago, USA

Professor Gönül O. Peker

Ege University School of Medicine

Izmir, Turkey

Professor David Prigollini

University of Buenos Aires

Buenos Aires, Argentina

\section{Dr. Pablo A. Pulido}

Executive Director

Panamerican Federation of Associations of Medical Schools

(PAFAMS), Caracas, Venezuela

Dr. Ebrahim M. Samba

Regional Director

World Health Organization

Regional Office for Africa

Harare, Zimbabwe

Professor Iskender Sayek

Hacettepe University

Ankara, Turkey

Dr. Mette Siemsen

Danish Medical Association

Copenhagen, Denmark

Dr. Nilanthi de Silva

University of Kelaniya

Ragama, Sri Lanka

\section{Dr. David P. Stevens}

Vice President, Association of American Medical Colleges (AAMC)

Washington, D.C., USA
Dr. Abu Bakar Suleiman

Director of Health

Ministry of Health

Kuala Lumpur, Malaysia

Dr. Jamsheer Talati

Associate Dean

The Aga Khan University

Karachi, Pakistan

\section{Dr. Cillian Twomey}

President, Union Européenne

des Médecins

Spécialistes (UEMS)

Cork, Ireland

\section{Professor Felix Vartanian}

Vice Rector

The Russian Academy of Advanced

Medical Studies

Moscow, Russian Federation

Theanne Walters

Deputy Executive Officer

Australian Medical Council

Canberra, Australia

\section{Dr. Dennis K. Wentz}

American Medical Association Chicago, USA

\section{Ass. Professor Ole Winding \\ World Federation for Medical \\ Education \\ Copenhagen, Denmark}

Dr. Gustaaf Wolvaardt

South African Medical Association Pretoria, South Africa 